\title{
The Militarization of Science, and Subsequent Criminalization of Scientists
}

\author{
Jaime A. Teixeira da Silva
}

Independent researcher

\section{CORRESPONDENCE \\ Jaime A. Teixeira da Silva \\ P. O. Box 7, Miki-cho post office, Ikenobe 3011-2, Kagawa-ken, 761- 0799, Japan \\ Tel/Fax: +81878988909 \\ E-mail: jaimetex@yahoo.com}

\section{ARTICLE HISTORY}

Received: 9 July, 2016

Accepted: 5 September, 2016

\begin{abstract}
Science is becoming more challenging, not only for scientists, but also for editors and publishers. Faced with limited funding within an expanding economic crisis, competition between scientists is increasing. The struggle for professional survival is leading some to revert to dishonest tactics to get ahead of the pack and cheating or fraud may be involved. Confronted with these new realities, which have become more debatable within the public arena, mainly as a result of an increase in blogs and social media, editors and publishers are reinforcing current publishing platforms in a bid to reduce the risks and to fortify their journals against future submission- and fraud-related problems. Ultimately, this places greater scrutiny - and stress - on the authorship, leading to an increase in militarization. At some point - which certain hints already indicate - the criminalization of science will begin as publishers fail to curtail fraud.
\end{abstract}

Keywords: accountability, criteria, post-publication peer review, responsibility, transparency

Science may be in much more than just a crisis of trust and reproducibility. Confronted by increasing awareness, expectations and competition for funding, scientists and their work are coming under increasing scrutiny. Long gone are the days when a manuscript could simply be submitted as an email attachment. And although such cases still exist, they are more the exception than the rule, with most journals now opting to use online submission systems (OSSs) that form part of a publishing management protocol, which ultimately involves a database that allows for backgrounds to be checked, publishing histories to be looked up, and suitable peers to be matched using matching keywords. At face value, one might argue that these OSSs increase the reliability of a submission and serve as the first line of verification of the authenticity of a submission. Indeed, this may be true, but the fact that scientists have no option but to comply with the requirements and conditions in an OSS - or submit their manuscript elsewhere if the task is too daunting - indicates that scrutiny, the first step in militarization, has become a norm in science publishing. This concept is not that far-fetched and is not the basis of some conspiracy theory about the publishing industry. Faced with increased cases of fake peer reviews, fake identities of authors, or other forms of fraud or misconduct that undermine the integrity of the submis- 
sion process, publishers are left with few options. Thus, to curb dishonest behavior, OSSs contain increasing checks and balances, and increasing number of check-boxes and requirements for statements that confirm the originality of a submission and that request detailed information about all authors, including their titles, institutional positions, and emails. These checks and balances constitute an additional layer of stress - except for authors who use third party services to complete online submissions - over and above the stress caused by conducting the research and completing peer review. Thus, although it is understandable that publishers are required to increase the number of verification steps for authors during the submission of a manuscript, it fortifies that the process is becoming stricter and more militarized, and not less.

The post-publication peer review movement, which involves an analysis of the already published literature after it has been published, usually by individuals not associated with that paper or with the original peer review of that paper, has added one new layer of scrutiny to scientists and the scientific literature. These efforts, coupled with additional pressure by critical science journalists, blogs and social media - which can elevate a seemingly innocuous error into a full-blown scandal within the matter of hours or days - add an additional layer of fear and scrutiny to the militarized state of science. In some cases, scientists try to fight back, seeking legal counsel in a bid to reverse retractions, but in almost all cases fail. Then of course, there is the anti-science movement that is skeptical and critical of all things that are science or sciencerelated. This sector of the public is extremely dangerous because it can use a simple error as ammunition against science or scientists. Thus, an error may be blown out of proportion, and what may in fact be an honest error, through puns and freedom of speech, can be portrayed as an act of misconduct, even if it is not. This vilification of error also is spurring a culture of counter-attack and defense, sometimes legal.
Thus, not only is there a veritable war taking place, not in terms of real bullets and land-mines, but in terms of attitudes and infrastructure, rules and regulations, that are limiting scientists' freedoms, that are curbing their confidence, and which is threatening to dismantle science. The increasing trend of making ORCID voluntary to making it obligatory, the fortification of iThenticate ${ }^{\bullet}$ and other forprofit plagiarism-detection software as the savior of science publishing's integrity by making it a formal part of the peer-review process, all indicate that science is on an irreversible course of militarization. The next logical step to militarization is criminalization. As more fraud is detected, as more errors get projected as misconduct, and as publishers increasingly shift the blame and responsibility upon the scientists' shoulders, it is natural that a population of "misfits" and side-lined researchers will begin to emerge. There will be a class of scientists that exhibit no publishing blemishes, and then there will be all others, those who have erred or who have errata or retractions to their names. Rather than embracing error as a natural evolutionary part of research and publishing, it is increasingly becoming demonized and vilified. And, as the anti-science rhetoric builds up, when coupled with skepticism fortified by proof from the literature, the movement to criminalize scientists, rather than to seek counsel and reform, is building up.

Most scientists will most likely agree that blatant fraud and misconduct are not acceptable. They will also likely agree that no such thing as perfect science exists, and that even the best of scientists will likely err. But there is genuine concern about the path that science publishing is taking by increasing the militarized stance, and that this will either mischaracterize true error, sideline genuinely motivated scientists who are willing to reform, or cause greater and irreparable damage to science's publishing foundations.

\section{CONFLICT OF INTEREST STATEMENT}

The author declares no conflicts of interest. 